\title{
Gas-liquid phase coexistence and finite-size effects in a two-dimensional Lennard-Jones system
}

\author{
OUYANG WenZe*, XU ShengHua \& SUN ZhiWei* \\ Key Laboratory of Microgravity (National Microgravity Laboratory), Institute of Mechanics, Chinese Academy of Sciences, Beijing 100190, China
}

Received September 8, 2010; accepted January 29, 2011

\begin{abstract}
The gas-liquid phase coexistence in a two-dimensional Lennard-Jones system is investigated using Maxwell construction method together with molecular dynamics simulations. The results of phase coexistence in different truncations of the potential are compared with data obtained from the literature, and the corresponding critical properties calculated. The crossover from Ising-like to mean field behavior is observed and confirmed as the temperature approaches the critical point from below. Performing simulations on systems with different sizes, we find that a finite size effect is more significant than those shown in most of the previous results, and a lower critical temperature is obtained when the full extent of this finite size effect is considered.
\end{abstract}

Lennard-Jones system, two dimension, gas-liquid phase coexistence, finite size effect, molecular dynamics

Citation: Ouyang W Z, Xu S H, Sun Z W. Gas-liquid phase coexistence and finite-size effects in a two-dimensional Lennard-Jones system. Chinese Sci Bull, 2011, 56: 2773-2779, doi: 10.1007/s11434-011-4611-Z

The phase behavior of two-dimensional (2D) systems continues to attract much attention from both experimental and theoretical points of view. Although a perfect 2D system does not exist in nature, it has practical relevance because many systems can be modeled as a $2 \mathrm{D}$ system, such as monolayers adsorbed on solid substrates, physisorption of gases on solid surface, and thinning of completely wetted films on clean mica surfaces.

There are a variety of techniques introduced to obtain phase coexistence properties [1-9], among which the Gibbs ensemble Monte Carlo (GEMC) method appears as the most popular because of its simplicity and efficiency [1]. Despite very impressive progress in recent years, there remain some problems and issues that need to be addressed. To efficiently obtain the phase coexistence properties of systems with complex molecular structures, some molecular dynamics (MD) methods have been proposed of late [2-4]. In three-dimensional (3D) systems, phase coexistence results from experiments, simulations, and theories are in reasonable agreement with each other. However, understanding

\footnotetext{
*Corresponding authors (email: oywz@imech.ac.cn; sunzw@imech.ac.cn)
}

gas-liquid (GL) phase coexistence and characterizing the related physical properties and precise location of the phase coexistence curve in 2D systems, even for simple fluids, still remains incomplete. The typical system, (i.e. 2D Lennard-Jones (LJ) fluid), has a very rough and foamy GL interface because of low surface tension, making it difficult to determine the GL phase coexistence curve. Among previous results from simulations of this $2 \mathrm{D}$ system, there is wide deviation in these curves.

Using the Gibbs ensemble technique, Singh et a1. [5,10] obtained the phase diagram and estimated the critical point of this 2D LJ system, with a critical temperature that was much lower than results from equation-of-state (EOS) fittings. Afterwards, Smit and Frenkel [11] revisited the GL phase coexistence of the same system. They improved the data and obtained a higher critical temperature as well as investigated the significant effect of the cutoff distance for the potential on the GL phase coexistence, an effect also occurring in 3D LJ systems [12]. Panagiotopoulos [13] studied the GL phase coexistence via GEMC in both 2D and 3D LJ systems. He observed that the phase coexistence of 2D LJ system had a crossover from Ising-like behavior to 
mean field behavior when the temperature approached the critical temperature from below, whereas such a crossover did not appear in 3D systems. Jiang and Gubbins [14] found that the resulting coexistence curve in GEMC for the adsorbed system was very close to that for the unperturbed 2D LJ film, and this suggested that experimental studies of such coexistence curves for simple systems could be approximately taken to be similar to those for an unperturbed 2D system. Meanwhile, they found that the results away from the critical temperature appeared to be essentially independent of the number of particles via GEMC simulations in systems of large size.

In this paper we adopt the Maxwell construction method together with MD simulations to investigate the GL phase coexistence in a 2D LJ system. Using different truncation potentials, the method involves obtaining the GL phase coexistence curves and critical properties which we then compare with other results from the literature. Furthermore, we perform simulations on different-sized systems to observe any finite-size effects on the GL phase coexistence.

\section{Model and simulation methodology}

The interacting potential in the $\mathrm{LJ}$ system is given by

$$
U_{\mathrm{LJ}}=4 \varepsilon\left[\left(\frac{\sigma}{r_{i j}}\right)^{12}-\left(\frac{\sigma}{r_{i j}}\right)^{6}\right],
$$

where $r_{i j}$ is the pair distance between the $i$ th and $j$ th particles. The parameter $\varepsilon$ governs the strength of the interaction, while $\sigma$ defines the length scale. For convenience, reduced units are used in simulations, where $\varepsilon, \sigma$, and the mass of $\mathrm{LJ}$ particle $m$ are chosen as basic units.

As conventionally adopted, the truncation of the interaction is used in the force calculation, as well as the periodic boundary conditions, and minimum image conventions are applied [15-17]. The LJ interactions decay rapidly with cutoff distance, thus a truncation correction is often made in calculations of thermodynamic quantities by adding a tail contribution, namely the so-called long-range correction. In Maxwell construction method, the focus is mainly on pressure. With the assumption that the radial distribution function $g(r) \approx 1$ when $r_{i j}$ is greater than the cutoff distance, the corresponding long-range correction to the pressure is

$$
P_{\text {tail }}=\frac{1}{2} \int_{r_{\text {cut }}}^{\infty} 2 \pi r \rho^{2} d r \frac{1}{2} \vec{r} \cdot \vec{f}(\vec{r})=\pi \rho^{2}\left(\frac{12}{5 r_{\text {cut }}^{10}}-\frac{3}{r_{\text {cut }}^{4}}\right),
$$

and for the potential

$$
U_{\text {tail }}=\frac{1}{2} \int_{r_{\text {cut }}}^{\infty} 2 \pi r \rho d r u(r)=\pi \rho\left(\frac{2}{5 r_{\text {cut }}^{10}}-\frac{1}{r_{\text {cut }}^{4}}\right) .
$$

Here $r_{\text {cut }}$ is the cutoff distance of the interaction truncation.
Note that the long-range correction for the 2D LJ system is different from that for the 3D case.

The MD simulations are performed in the canonical (or NVT) ensemble, i.e. the total number of particles $N$, the temperature $T$, and the volume of simulation cell $V$ are kept constant. The equation of motion for the particles is integrated using the velocity Verlet algorithm with time step $\delta t=$ 0.002 and temperature controlled via a Berendsen thermostat [18]. For a typical NVT simulation, the procedure is as follows: Initially, the $N$ particles are homogeneously put into the simulation cell separately. Their initial positions are on a square lattice and their initial velocities satisfy the Maxwell-Boltzmann distribution. Next, the system is heated to a higher temperature $\left(T>T_{\mathrm{c}}\right)$ so that the initial configuration has completely melted. Subsequently, the configuration is cooled down to the desired temperature and allowed to relax for some time steps so as to reach equilibrium. Finally, another long run is initiated and the calculated values of various physical properties are recorded every few time steps to obtain average values.

The GL phase coexistence curves are obtained via the Maxwell construction method, so a set of NVT simulations are performed along an isotherm. In the present work, the set of simulations was started from a very dilute gas phase $(\rho=0.001)$, and went through a typical NVT process. Next, the simulation cell was compressed to a smaller volume and another NVT simulation began. Note that the initial configuration of the next simulation is obtained directly from the previous configuration so that there is no need to initialize the configuration again. By repeating this procedure until a large enough density or a small enough volume needed for the Maxwell construction is reached, ample data relating pressure to volume are collected. To eliminate hysteresis effects, procedures are reversed, i.e. expansion is started immediately after compression is finished. By fitting the data for a series of temperatures, we can obtain the GL phase coexistence curve.

Figure 1 is a typical example of Maxwell construction fitting, which allows us to obtain the coexisting densities for pure gas and pure liquid. Due to finite-size effects, the $P-T$ phase-diagram results from computer simulations can appear similar to mean field predictions, so we can treat the van der Waals loop by fitting a mean field equation. Specifically, we fit the isotherm data for which the compressibility is positive using a modified van der Waals equation of state [19]

$$
P=\frac{N T}{V-N a_{1}}-\frac{a_{0}}{\left(V-N a_{2}\right)\left(V+N a_{3}\right)},
$$

where $a_{i}(i=0,1,2,3)$ are adjustable parameters. The circle and the square symbols correspond respectively to compression and subsequent expansion along an isotherm. Note that the logarithm is taken on the $x$-axis (volume) for a clearer view of the plots. 


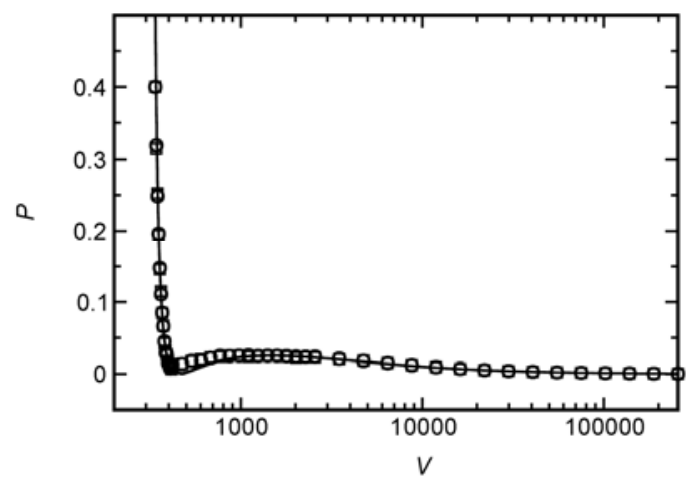

Figure 1 A typical curve using the Maxwell construction at $T=0.5$ and $r_{\text {cut }}=L / 2$ together with long-range correction. Open circles (squares) represent $P-V$ results obtained under compression (expansion). The solid line is the Maxwell construction fitting using eq. (4).

\section{Results and discussion}

The truncation of the interaction has a significant effect on the GL phase coexistence in both 2D and 3D systems. To be able to compare conveniently our results with those found in the literature, we used three typical cutoff distance values: $r_{\text {cut }}=2.5, r_{\text {cut }}=5.0$, and $r_{\text {cut }}=L / 2$. The number of particles is typically $N=256$. Note that the long-range correction is only applied for $r_{\text {cut }}=L / 2$ (see eqs. (2) and (3)) so that the full potential is represented.

Results for the GL phase coexistence of the 2D LJ system are plotted in Figure 2. As reported in the literature, the well-known scaling relations are usually adopted to estimate the critical temperature $T_{\mathrm{c}}$ and critical density $\rho_{\mathrm{c}}[17]$ :

$$
\rho_{L}-\rho_{G}=A\left|T-T_{c}\right|^{\beta},
$$

and

$$
\left(\rho_{L}+\rho_{G}\right) / 2=\rho_{c}+B\left|T-T_{c}\right| .
$$

As confirmed empirically in GEMC simulations, the phase coexistence curves can be fitted with an exponent $\beta=$ 0.125 (Ising value) over a wide range of temperatures. Some years ago, Panagiotopoulos [13] used GEMC to observe a clear crossover from Ising-like $(\beta=0.125)$ to mean field $(\beta=0.5)$ behavior as the temperature approached the critical point from below. For the $2 \mathrm{D}$ LJ system, the order parameter can be defined as

$$
M(T)=\rho_{L}-\rho_{G} .
$$

In Figure 3, we plotted the results of $M^{2}$ and $M^{8}$ versus $T$ with different interaction truncations. To ensure the values of $M^{8}$ and $M^{2}$ have approximately the same numerical range, the $M^{8}$ curves are shifted up by multiplying by 5 . In all cases, the exponent $\beta$ has a value of 0.125 when the temperature $T$ is away from the critical temperature $\left(T_{\mathrm{c}}\right)$ and approaches 0.5 when $T$ is close to $T_{\mathrm{c}}$ (see the linear fitting line in Figure 3), in accord with observations reported in
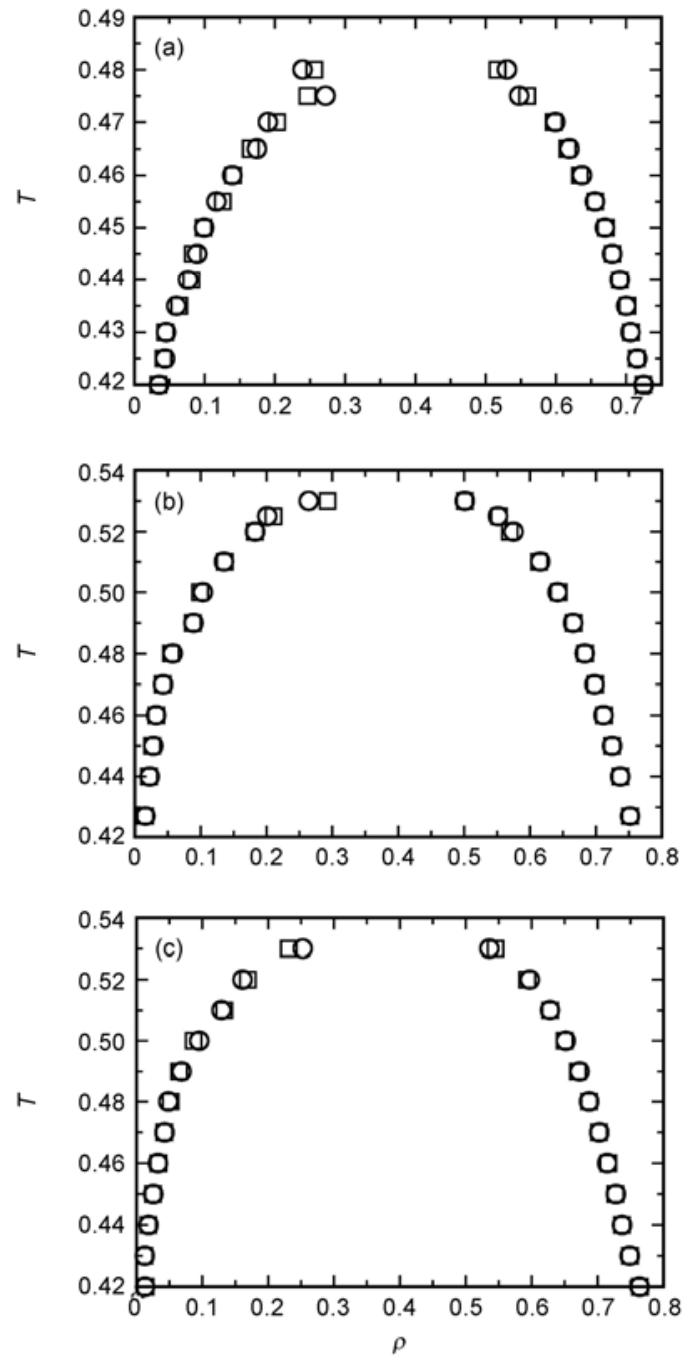

Figure 2 Gas-liquid phase coexistence using different interaction truncations. The number of particles is $N=256$. $r_{\text {cut }}=2.5$ (a), 5.0 (b), $L / 2$ (c). Note that the long-range correction is only applied for $r_{\text {cut }}=L / 2$. Open circles (squares) represent results obtained under compression (expansion).

ref. [13].

The estimates of $T_{\mathrm{c}}$ can be obtained by linearly fitting the curve of $M^{8}$ versus $T$. Apparently the intercept of the fitting line on $T$ axis is exactly the critical temperature $T_{\mathrm{c}}$ (Figure 3 ). The value of $T_{\mathrm{c}}$ for all cases was estimated by this procedure, and the corresponding critical densities $\rho_{\mathrm{c}}$ are fitted using eq. (6). The results of these critical points are listed in Table 1 where other critical properties (including critical pressure $P_{\mathrm{c}}$ and critical compressibility factor $\left.P_{\mathrm{c}} /\left(T_{\mathrm{c}} \rho_{\mathrm{c}}\right)\right)$ are also calculated using long runs of NVT simulations at the estimated critical points.

From Table 1, when the cutoff distance $r_{\text {cut }}$ is increased from 2.5 to 5.0 , the critical temperature $T_{\mathrm{c}}$ increases correspondingly from 0.457 to about 0.5 . In particular, when the cutoff distance approaches a large enough value, $r_{\text {cut }}=5.0$, the results are almost the same as those of the full potential $\left(r_{\text {cut }}=L / 2\right.$ plus long-range correction), which means that the effect of truncation on the GL phase coexistence is slight or 

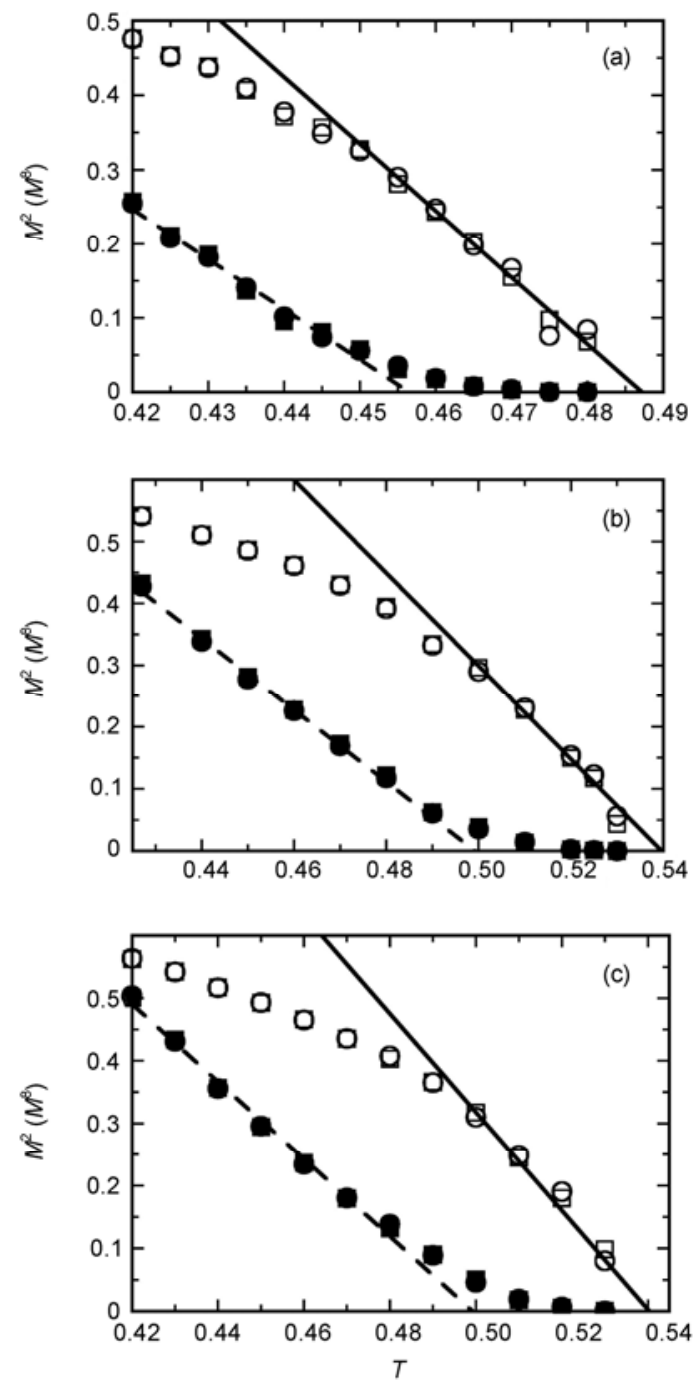

Figure 3 Curves of order parameter $M$ versus $T$. Open circles (squares) represent results of $M^{2}$ versus $T$ obtained under compression (expansion). The number of particles is $N=256 . r_{\text {cut }}=2.5$ (a), 5.0 (b), $L / 2$ (c). Solid circles (squares) represent results of $M^{8}$ versus $T$ obtained under compression (expansion). The solid (dashed) line denotes linear fitting in the region of mean field (Ising-like) behavior. even negligible. Santra et al. [20] obtained similar converging results; they observed that the gas and liquid coexistence densities at a certain temperature remained unchanged for large enough cutoff distance. This can be explained by eqs. (2) and (3). As is well-known, the GL phase coexistence at a desired temperature is determined by the equilibrium of both pressure and chemical potential. Assuming that $r_{\text {cut }}=5.0$, we obtain $P_{\text {tail }}=0.0151 \rho^{2}$ and $\mu_{\text {tail }}=2 U_{\text {tail }} \approx$ $0.0101 \rho$, the latter being the long-range correction of the chemical potential. Compared with the total value and statistical uncertainties of $P$ and $\mu$, such long-range correction is small enough as to be negligible when $r_{\text {cut }}>5.0$. In the 3D LJ system, the long-range correction has a relatively larger value and is approximately proportional to $r_{\text {cut }}^{-3}$ while the long-range correction in the $2 \mathrm{D} \mathrm{LJ}$ system is approximately proportional to $r_{\text {cut }}^{-4}$.

It is worthwhile comparing our estimated critical points with available literature data. To begin, let us focus on the case $r_{\text {cut }}=2.5$. Rovere et al. [6,21] have suggested a critical temperature $T_{\mathrm{c}}=0.5$, afterwards giving an improved estimate, i.e. $T_{\mathrm{c}}=0.472$ and $\rho_{\mathrm{c}}=0.35$. Smit and Frenkel [11] performed GEMC simulations for the 2D LJ system, and obtained a lower critical temperature value $T_{\mathrm{c}}=0.459$ together with $\rho_{\mathrm{c}}=0.35$. Using data from GEMC simulations and an Ising exponent to fit the phase coexistence curve away from the critical point, Panagiotopoulos [13] obtained $T_{\mathrm{c}}=0.477$ and $\rho_{\mathrm{c}}=0.38$. Our results of critical temperature $T_{\mathrm{c}}=0.457$ is closer to the Smit-Frenkel result except for a slightly higher critical density. Actually, the estimate of the critical temperature depends sensitively on the data collected just below the critical point. In Figure 3, one can see that the system has a mean field behavior in a wide range of temperatures from $T=0.45$ to $T=0.48$. Previously, authors empirically claimed that the phase coexistence curves could be fitted over a wide range of temperatures using the Ising value in $2 \mathrm{D}$ systems. As our phase coexistence data is not well-fitted by eq. (5) with Ising value $\beta=0.125$, we have

Table 1 Critical point and critical properties for Ising-like behavior and mean field behavior using different interaction truncations. The number of particles is $N=256$. The compressibility factor is defined as $\tau_{\mathrm{c}}=P_{\mathrm{c}} /\left(T_{\mathrm{c}} \rho_{\mathrm{c}}\right)$. Note that the long-range correction is applied for $r_{\text {cut }}=L / 2$ so that the full potential is represented

\begin{tabular}{|c|c|c|c|c|c|c|c|c|}
\hline \multicolumn{9}{|c|}{ Ising-like behavior fitting } \\
\hline \multirow{2}{*}{$r_{\mathrm{cut}}$} & \multicolumn{4}{|c|}{ Compression } & \multicolumn{4}{|c|}{ Expansion } \\
\hline & $T_{\mathrm{c}}$ & $\rho_{\mathrm{c}}$ & $P_{\mathrm{c}}$ & $\tau_{\mathrm{c}}$ & $T_{\mathrm{c}}$ & $\rho_{\mathrm{c}}$ & $P_{\mathrm{c}}$ & $\tau_{\mathrm{c}}$ \\
\hline 2.5 & 0.457 & 0.386 & 0.021 & 0.119 & 0.457 & 0.386 & 0.0209 & 0.1185 \\
\hline 5.0 & 0.499 & 0.36 & 0.0246 & 0.1366 & 0.5 & 0.36 & 0.0241 & 0.1341 \\
\hline$L / 2$ & 0.501 & 0.363 & 0.0239 & 0.1315 & 0.5 & 0.365 & 0.0231 & 0.1268 \\
\hline \multirow{2}{*}{$r_{\mathrm{cut}}$} & \multicolumn{4}{|c|}{ Compression } & \multicolumn{4}{|c|}{ Expansion } \\
\hline & $T_{\mathrm{c}}$ & $\rho_{\mathrm{c}}$ & $P_{\mathrm{c}}$ & $\tau_{\mathrm{c}}$ & $T_{\mathrm{c}}$ & $\rho_{\mathrm{c}}$ & $P_{\mathrm{c}}$ & $\tau_{\mathrm{c}}$ \\
\hline 2.5 & 0.487 & 0.407 & 0.0363 & 0.1832 & 0.487 & 0.408 & 0.0367 & 0.1849 \\
\hline 5.0 & 0.54 & 0.38 & 0.044 & 0.2149 & 0.538 & 0.381 & 0.0429 & 0.2096 \\
\hline$L / 2$ & 0.542 & 0.396 & 0.0441 & 0.2051 & 0.541 & 0.394 & 0.0429 & 0.2013 \\
\hline
\end{tabular}


only taken the region of Ising-like behavior to obtain the critical point omitting the region $T>0.45$. This is probably the reason for the difference between our results and those in the literature.

With a full potential ( $r_{\text {cut }}=L / 2$ plus long-range correction), it is already clear that the estimate of $T_{\mathrm{c}}=0.472$ by Singh et al. is too low, while our results are in fairly good agreement with GEMC simulations [10,11,13,14,22]. Specifically, Jiang and Gubbins [14] performed GEMC simulations on several large-sized systems; essentially, their results appeared to be independent of $N$. It should be mentioned that the critical points we obtain here (Table 1) are in excellent agreement with Jiang and Gubbins' results, $T_{\mathrm{c}}=$ $0.498 \pm 0.002$ and $\rho_{\mathrm{c}}=0.36 \pm 0.005$. Figure 3 shows that Ising-like behavior predominates over a wide range from low temperature to $T \approx 0.48$ and mean field behavior predominates only above $T=0.5$, a feature that is quite different if $r_{\text {cut }}=2.5$. Therefore, it is no surprise that the critical point can be estimated very well.

Away from the critical point, the system can accommodate all relevant fluctuations and exhibit Ising-like behavior. Close to the critical point, the system is too small to accommodate every fluctuation because the correlation length increases notably exceeding the size of the simulation box, and, as a consequence, mean field behavior is observed. The phase coexistence for finite-size systems can be observed at temperatures higher than the estimated critical point (Figures 2 and 3), which implies significant finite-size effects. Next, let us explain why results reported in some of the literature are higher than our estimates. If we consider finite systems, especially small systems, and try to estimate the critical point, the data close to the critical point must be included. To fit the phase coexistence region close to $T_{\mathrm{c}}$ where the system presents the mean field behavior, a dif- ferent critical point, specifically a higher $T_{\mathrm{c}}$, can be achieved. The results are also compiled in Table 1. As is well-known, the results from equations of state (EOS) also have a higher $T_{\mathrm{c}}$. For instance, Reddy and O'Shea (RO) [5] used EOS together with additional data from conventional Monte Carlo simulations $\left(r_{\text {cut }}=L / 2\right.$, with long-range correction) and the viral EOS to fit a 33 parameter BenedictWebb-Rubin equation. From the EOS, they obtained an estimate for the critical point of $T_{\mathrm{c}}=0.537$ and $\rho_{\mathrm{c}}=0.365$. It seems that the estimated $T_{\mathrm{c}}$ via EOS is in good agreement with our results, viz. $T_{\mathrm{c}}=0.54$ estimated by fitting the region of mean field behavior. Such good agreement is, however, somewhat exaggerated, because the EOS in ref. [5] seems to have an artifact and exhibits a strange hump close to the critical point where the mean field behavior has been previously investigated. Some years ago, Mulero et al. [23] gave the data of GL phase coexistence from the semi-empirical RO EOS. For comparison, their results are listed in Table 2 together with our results for the full potential $\left(r_{\text {cut }}=\mathrm{L} / 2\right.$ plus long-range correction). The phase coexistence obtained from RO EOS is in excellent agreement with our Maxwell construction results at low temperature. When the temperature is increased, the deviation between RO EOS data and our results becomes increasingly significant. Furthermore, when we try to calculate the order parameter $M^{2}$ and $M^{8}$ using the data of EOS, the crossover from Ising-like behavior to mean field behavior is not found at all via the relation of $M$ versus $T$.

As is well-known, the van der Waals loops in simulations are due to finite-size effects and may become weaker for systems with large particle numbers [24]. To further explore finite-size effects in the 2D LJ system, we perform another set of simulations using the Maxwell construction in systems where $N=144,196,324,400,1024$. We begin by

Table 2 Gas-liquid phase coexistence at full potential $\left(r_{\text {cut }}=L / 2\right.$ together with long-range correction)

\begin{tabular}{|c|c|c|c|c|c|c|}
\hline \multirow[b]{2}{*}{$T$} & \multicolumn{2}{|c|}{ Ref. [23] } & \multicolumn{2}{|c|}{ This paper (compression) } & \multicolumn{2}{|c|}{ This paper (expansion) } \\
\hline & $\rho_{\mathrm{G}}$ & $\rho_{\mathrm{L}}$ & $\rho_{\mathrm{G}}$ & $\rho_{\mathrm{L}}$ & $\rho_{\mathrm{G}}$ & $\rho_{\mathrm{L}}$ \\
\hline 0.42 & 0.01776 & 0.75532 & 0.01311 & 0.7637 & 0.01405 & 0.76399 \\
\hline 0.43 & 0.02109 & 0.74685 & 0.01266 & 0.74879 & 0.01242 & 0.74908 \\
\hline 0.44 & 0.02494 & 0.73755 & 0.01874 & 0.73738 & 0.01798 & 0.73695 \\
\hline 0.45 & 0.0294 & 0.72752 & 0.02577 & 0.72799 & 0.02525 & 0.7271 \\
\hline 0.46 & 0.0346 & 0.71677 & 0.03287 & 0.71484 & 0.0333 & 0.71609 \\
\hline 0.47 & 0.0407 & 0.70521 & 0.04284 & 0.70283 & 0.04238 & 0.70212 \\
\hline 0.48 & 0.04794 & 0.69262 & 0.0487 & 0.68722 & 0.05201 & 0.68703 \\
\hline 0.49 & 0.05672 & 0.67859 & 0.06849 & 0.6726 & 0.06484 & 0.66996 \\
\hline 0.5 & 0.06772 & 0.66236 & 0.09541 & 0.65181 & 0.08678 & 0.65004 \\
\hline 0.51 & 0.08241 & 0.6422 & 0.12938 & 0.62791 & 0.13335 & 0.62819 \\
\hline 0.52 & 0.10504 & 0.61268 & 0.1611 & 0.59728 & 0.16921 & 0.59323 \\
\hline 0.53 & 0.23368 & 0.48612 & 0.25263 & 0.5358 & 0.23151 & 0.54528 \\
\hline
\end{tabular}


studying the conventional case with $r_{\text {cut }}=2.5$. The curves of the estimated critical temperature in the region of Ising-like behavior $T_{\mathrm{c}}$ versus $N$ are shown in the top panel of Figure 4 (including our previous result with $N=256$ ). The curve corresponding to critical density versus system size is not presented because no clear trend is seen due to statistical uncertainties. Looking at the figure, the estimated $T_{\mathrm{c}}$ is dependent on the size of system, i.e. the $T_{\mathrm{c}}$ of larger systems are obviously lower than those of smaller systems. This is different from the results obtained in GEMC simulations $[11,13]$, where finite-size effects are much smaller and often approximately negligible. In GEMC simulations, the total volume of two simulation boxes is kept constant but the size of each box fluctuates, so the system size can only be controlled by varying the number of particles and initial densities of the two boxes together with the preliminary estimates of the equilibrium densities for each system and temperature studied. However, in a typical set of Maxwell construction simulations, volumes of the simulation boxes are varied during compression or expansion procedures; therefore the system size is actually not constant and not comparable to that in GEMC simulations. Strictly speaking, the system size during our simulations is not constant either because the volume is varied relatively by changing the number of particles. It is probably for this reason why our results of finite size effect are so different from those obtained via
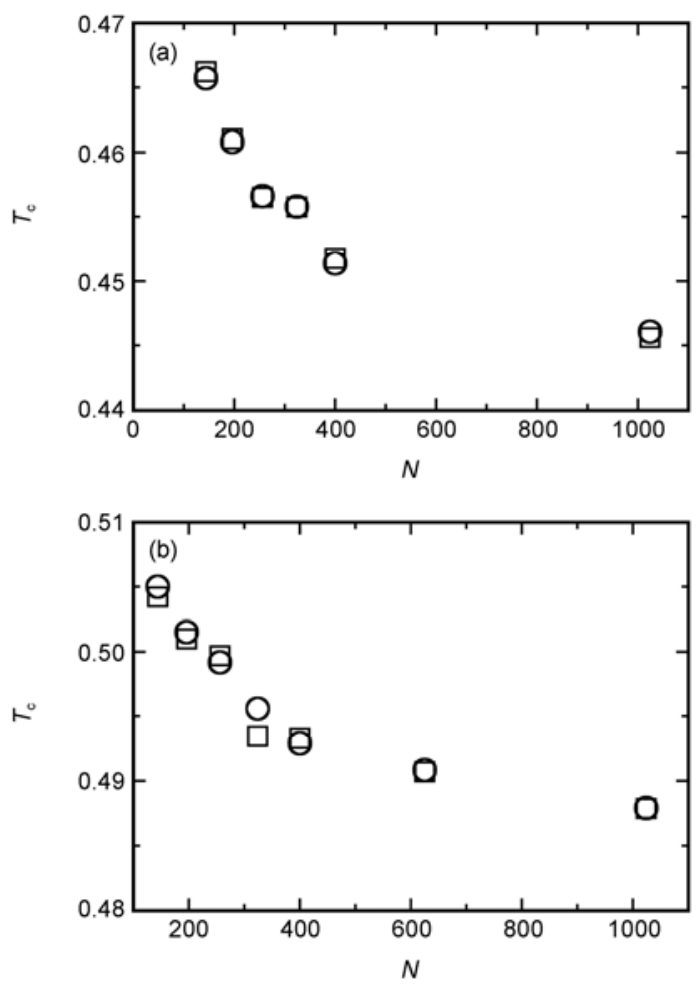

Figure 4 Particle number $(N)$ dependence for the estimated critical temperature $T_{\mathrm{c}}$ in the region of Ising-like behavior. (a) $r_{\mathrm{cut}}=2.5$; (b) full potential. Open circles (squares) represent results obtained under compression (expansion).
GEMC. Indeed there are some previous results of finite-size effects which are in agreement with our present observation. For instance, we recently used the particle-transfer MD simulation method to construct the phase coexistence curve of the 3D LJ system, and observed that the results on finite-size effects were also different from GEMC results [4]. Wilding and Bruce [25,26] even reported an extensive MC study of the density and energy fluctuations in 2D LJ fluids within the grand-canonical ensemble, and the results are analyzed using finite-size-scaling theory. Their study lead to $T_{\mathrm{c}}=0.440 \pm 0.005$. Note that their cutoff distance for the potential truncation is $r_{\text {cut }}=2.0$, so the obtained critical temperature should be slightly lower than for $r_{\text {cut }}=2.5$. Nevertheless, their result is in perfect accord with our present result (Figure 4(a)).

Furthermore, we investigated the case of full potential with different system sizes. The curve for the estimated $T_{\mathrm{c}}$ versus $N$ is plotted in Figure 4(b). From the tendency of the curve, we can obtain an approximate value of the critical temperature for the system with full potential, i.e. $T_{\mathrm{c}} \approx 0.485$, which is also smaller than previous results $[11,13,14]$.

In general, we note that most previously estimated $T_{\mathrm{c}}$ values for the $2 \mathrm{D} \mathrm{LJ}$ system are too high when considering the full extent of finite-size effects.

\section{Conclusion}

In this paper, we described the use of the Maxwell construction method together with MD simulations in the investigation of GL phase coexistence in a 2D LJ system. The critical points were estimated and the corresponding critical properties calculated. Differences between our results and those found in the literature were analyzed in detail. In agreement with previous GEMC results, the estimated critical temperature depended significantly on the truncation method of the LJ potential, although the critical density showed no clear trend as a result of statistical errors. Moreover, when the cutoff distance approached sufficiently large values, the GL phase coexistence curve and estimated critical point are identical to those of the full potential.

The finite-size effects in the $2 \mathrm{D} \mathrm{LJ}$ system were further studied. Via order parameter versus temperature fitting both in the region away from the critical point and in the vicinity of the critical point, the crossover phenomenon from Isinglike behavior to mean-field behavior, was observed and confirmed. Our simulation data indicated that the estimated critical temperatures are largely dependent on system size, in contradistinction to previous GEMC simulation results. When the full extent of finite-size effects is considered, we observed an estimated critical temperature of $T_{\mathrm{c}} \approx 0.485$, which is evidently lower than most of reported results, i.e. most estimated critical temperatures found in the literature are probably too high. 
This work was supported by the National Natural Science Foundation of China $(20903112,10972217)$ and the Knowledge Innovation Program of Chinese Academy of Sciences (KJCX2-YW-LO8).

1 Panagiotopoulos A Z. Direct determination of phase coexistence properties of fluids by Monte-Carlo simulation in a new ensemble. Mol Phys, 1987, 61: 813-826

2 Moller T, Fischer J. Vapor-liquid-equilibrium of a pure fluid from test particle method in combination with NPT molecular-dynamics simulations. Mol Phys, 1990, 69: 463-473

3 Palmer B J, Lo C M. Molecular dynamics implementation of the Gibbs ensemble calculation. J Chem Phys, 1994, 101: 10899-10907

4 Ouyang W Z, Lu Z Y, Shi T F, et al. A molecular-dynamics simulation study on the dependence of Lennard-Jones gas-liquid phase diagram on the long-range part of the interactions. J Chem Phys, 2005, 123: 234502

5 Reddy M R, O'Shea S F. The equation of state of the two-dimensional Lennard-Jones fluid. Can J Phys, 1986, 64: 677-684

6 Rovere M, Heermann D W, Binder K. The gas-liquid transition of the two-dimensional Lennard-Jones fluid. J Phys Condens Matter, 1990, 2: 7009-7032

7 Conde M M, Vega C. Determining the three-phase coexistence line in methane hydrates using computer simulations. J Chem Phys, 2010, 133: 064507

8 Zeng X C. Gas-liquid nucleation in two-dimensional fluids. J Chem Phys, 1996, 104: 2699-2704

9 Jia R, Hentschke R. Dipolar particles in an external field: Molecular dynamics simulation and mean field theory. Phys Rev E, 2009, 80: 051502

10 Singh R R, Pitzer K S, De Pablo J J, et al. Monte Carlo simulation of phase equilibria for the two-dimensional Lennard-Jones fluid in the Gibbs ensemble. J Chem Phys, 1990, 92: 5463-5466

11 Smit B, Frenkel D. Vapor-liquid equilibria of the two-dimensional Lennard-Jones fluid(s). J Chem Phys, 1991, 94: 5663-5668

12 Smit B. Phase diagrams of Lennard-Jones fluids. J Chem Phys, 1992,
1296: 8639-8640

13 Panagiotopoulos A Z. Molecular simulation of phase coexistence: Finite-size effects and determination of critical parameters for twoand three-dimensional Lennard-Jones fluids. Int J Thermophys, 1994, 15: $1057-1072$

14 Jiang S, Gubbins K E. Vapor-liquid equilibria in two-dimensional Lennard-Jones fluids: Unperturbed and substrate-mediated films. Mol Phys, 1995, 86: 599-612

15 Rappaport D C. The Art of Molecular Dynamics Simulation. 2nd ed. Cambridge: Cambridge University Press, 1997

16 Allen M P, Tildesley D J. Computer Simulation of Liquids. Oxford: Clarendon Press, 1987

17 Frenkel D, Smit B. Understanding Molecular Simulation: From Algorithms to Applications. 2nd ed. San Diego: Academic Press, 1996

18 Berendsen H J C, Postma J P M, van Gunsteren W F, et al. Molecular dynamics with coupling to an external bath. J Chem Phys, 1984, 81: 3684-3690

19 Wei Y S, Sadus R J. Equation of state for the calculation of fluidphase equilibria. AIChE J, 2000, 46: 169-196

20 Santra M, Chakrabarty S, Bagchi B. Gas-liquid nucleation in a two dimensional system. J Chem Phys, 2008, 129: 234704

21 Rovere M, Nielaba P, Binder K. Simulation studies of gas-liquid transitions in two dimensions via a subsystem-block-density distribution analysis. Z Phys B Condens Matter, 1993, 90: 215-228

22 Glandt E D, Fitts D D. Percus-Yevick equation of state for the twodimensional Lennard-Jones fluid. J Chem Phys, 1977, 66: 4503-4508

23 Mulero A, Cuadros F, Faundez C A. Vapor-liquid equilibrium properties for two- and three-dimensional Lennard-Jones fluids from equations of state. Aust J Phys, 1999, 52: 101-115

24 Yamamoto R, Kitao O, Nakanishi K. Can the 'van der Waals loop' vanish? II. Effect of domain size. Mol Phys, 1995, 84: 757-768

25 Bruce A D, Wilding N B. Scaling fields and universality of the liquidgas critical-point. Phys Rev Lett, 1992, 68: 193-196

26 Wilding N B, Bruce A D. Density-functions and field mixing in the critical fluid. J Phys Condens Matter, 1992, 4: 3087-3108

Open Access This article is distributed under the terms of the Creative Commons Attribution License which permits any use, distribution, and reproduction in any medium, provided the original author(s) and source are credited. 